\title{
Simulation and Implementation of 2-order Chebyshev Chaotic Sequence Based on FPGA
}

\author{
Tao Wang, Liyong Bao \\ School of Information Engineering, Yunnan University, Yunnan Province, China \\ bly.yx@163.com, lybao@ynu.edu.cn
}

\begin{abstract}
The spread spectrum communication, as one of the most important techniques in the 21 st century, receives a universal attention to the research on its key techniques. To study the performances of the chebyshev chaotic sequence in digital communication, mathematical analysis is made on the autocorrelation and cross-correlation property, and this paper gives a digital implementation of chebyshev chaotic sequence which is based on FPGA. The chebyshev chaotic sequence is generated by bit quantification function in quartus. It also has randomness and good correlation. The experimental results demonstrate that the output of the chebyshev chaotic sequence is stable and the advantages of without environment interference in the DE2.

Index Terms - spread spectrum communication, Chebyshev chaotic sequence, the autocorrelation and cross-correlation property, quantization, FPGA
\end{abstract}

\section{Introduction}

Chaotic motion is contains the disorder in an orderly, certainty in reflecting the probabilistic similar to random movement [1-5]. It is sensitive to initial value. A tiny change can bring a completely different phenomenon. Therefore, this kind of phenomenon is known as the "butterfly effect". Because of the chaotic movement with the bounded within a certain range, randomness, it is more and more get people's attention in the field of communication and become a research hot spot. The spread spectrum communication technology has far cannot meet the demand of people's needs for communication security, especially in the field of military communication security requirements. Certainty and roundedness can enable people to control the transmission of the communication. Disorder, randomness and irreversibility of transmission will enhance the security of communication, so as to meet the demand of the safety of the civilian and military. Sensitivity to the initial value, it can expand the scope of its use[6,7]. But analog circuit implementation of chaotic systems has high cost and vulnerable to environment which leads to the formation of a perturbation cycle state[7]. In this paper, the advantages of using FPGA is not influenced by external environmental factors, we put forward the digital implementation scheme of second- order chebyshev chaotic sequence based on FPGA. Firstly, in quartus, the use of 32 bit digital quantization of second-order chebyshev chaotic sequence conducts a waveform simulation. Then, download it to the DE2 operated and we will observe the results. Ultimately, we can observe 2-order chebyshev chaotic sequence data in hexadecimal stable output in DE2.

\section{Chebyshev Chaotic Analysis and Simulation}

Now often uses chebyshev, logistic and other chaotic sequences. Among them, the logistic's study has been more mature. In the literature [3], analyzes the characteristic of the improved logistic, and the mean value is 0.5 . In the literature [5] gives the chebyshev mean value of 0.5 .

The chebyshev expressions:

$$
x_{n+1}=\cos \left(k * \cos ^{-1} x_{n}\right),-1<x_{n}<1, n=1,2, \ldots
$$

When the value of $x_{n}$ between $(1,1)$ and $k$ is even, after iterations can generate chaotic sequence.

The Logistic mapping expressions:

$$
x_{n+1}=u * x_{n}\left(1-x_{n}\right), 0<x_{n}<1, n=1,2, \ldots
$$

In this expression, $1 \leq u \leq 4$. When $u=4$, the system is in full mapping. Therefore, when $k=2$, initial value $x_{1}=0.2$, we first simulate chebyshev chaotic sequence iteration times 1024, it will generate second-order real numerical sequence $[3,4]$.

Fig. 1 and Fig. 2 give the real value sequence of chebyshev which is chaotic sequence. The more the times of iterations, chaotic phenomenon is more obvious. Because chebyshev real value chaotic sequence has good autocorrelation and cross-correlation, so it could be applied in future communication. However, we must be the real quantitative numerical sequence to convenient for our use, and also need to test whether our numerical quantitative still meet its real numerical characteristics of the chaotic sequence is presented.

\section{Sequences Numerical Binary Quantization}

Now commonly used digital quantitative methods are: threshold quantization, symmetric binary quantitative, quantitative, quantitative extraction, mean quantization bits. In this article choose bits of extracting quantitative method.

Bits of quantitative extraction is to quantitative $L$ bits of iteration point, make each iteration point to produce a length $L$ of the binary. The expression:

$$
X_{n}=x_{n} * 2^{L}, X_{n}=\sum_{i=1}^{L} a_{i} * 2^{L-i}
$$

In literature [2] discusses two aspects: A method is given different initial values produce different chaotic sequence, and $L$ bit quantized respectively; the other method is to quantify the given an initial value to generate chaos sequence, then draw $L$ bit according to certain intervals. No matter what kind of method is used the mean value is $0.5[3,5]$. Therefore, in this 
article, the second way is adopted to chebyshev chaotic sequence for binary quantization. When $k=4, x_{1}=0.2$, we use threshold quantization and bit extraction quantization to observe the characteristics of chebyshev quantized. With the method of quantitative $L$ bits, the resulting binary chaotic sequence theory to $2 L$ bits. By the result of [2], we know when $L=32$, the quantitative characteristic of logistic is better. Therefore, we also adopt the $L=32$ bit to explore chebyshev sequence features.

Fig. 3 and Fig. 4 show the chebyshev bits of extracting quantitative also still keep the characteristics of the sequence of its real value, so the quantitative performance meets our requirements. Because the computer only discern the 0 or 1 , and quantify the value of the real value will be converted into 0 or 1 . This will help to chaotic sequence in the application of the communication system as early as possible.

\section{The Hardware Realization of Chebyshev}

Has the 32 bits quantization of chebyshev chaotic the randomness, security, initial value sensitivity, boundedness and other related features? This article through the DE2 hardware testing binary quantization conforms to its features. In the literature [6], with $L=32$ bits quantization for hardware implementation, but as a result of DE2 limited resources as well as the size of the display screen can only realize 2-order chebyshev chaotic sequence. In order to study the 4-order, 8-order and higher order chebyshev chaotic sequence, we must search for a better implementation scheme and principle.

The lcd1602 has eleven instructions. According to the lcd1602 instructions and in accordance with the 32 bit binary output into 8 hexadecimal output, we can realize the output of 4-order.

Fig. 5 and Fig. 6 give the principle diagram of hardware implementation and waveform simulation. In the literature [5] has given the generating principle of four order chebyshev chaotic binary structure. Quartus waveform simulation is according to the principle diagram, part of it as shown in Fig. 6.

But in order to extract more directly to observe bit quantitative 32 bit chebyshev chaotic sequence, we need to implement on the DE2. Here, the specific external frame diagram is as follows:

Fig. 7 is a module diagram which is generated in the quartus. The reference literatures [8], [9] and [10] in Verilog HDL language usage and Alter the use of FPGA manual, use frequency meter, counters, state machine, etc. When the chebyshev chaotic sequence every time the 32 bit binary output, taken from low to high every four into a set of corresponding display a hexadecimal number. The realization of the lcd1602 display is according to each input a hexadecimal data, then using the initialization, reset, shift according to such a state machine. Specially needs to be pointed out is about frequency settings.

Fig. 8 shows the output on the DE2 32 bit binary data into hexadecimal. By looking at the data on the display, we can verify the previous bits quantization is correct. And using hexadecimal, can effectively avoid the display screen is not big enough can't display 48, 64 bit decimal quantification.

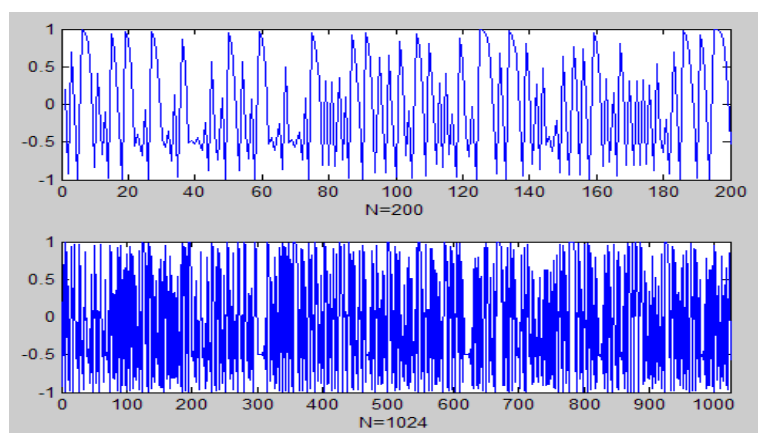

Fig. 1 Chebyshev sequence

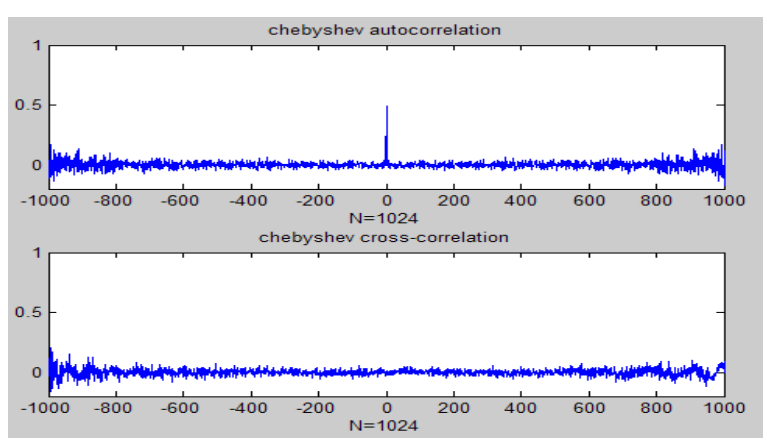

Fig. 2 Chebyshev auto-correlation and cross-correlation

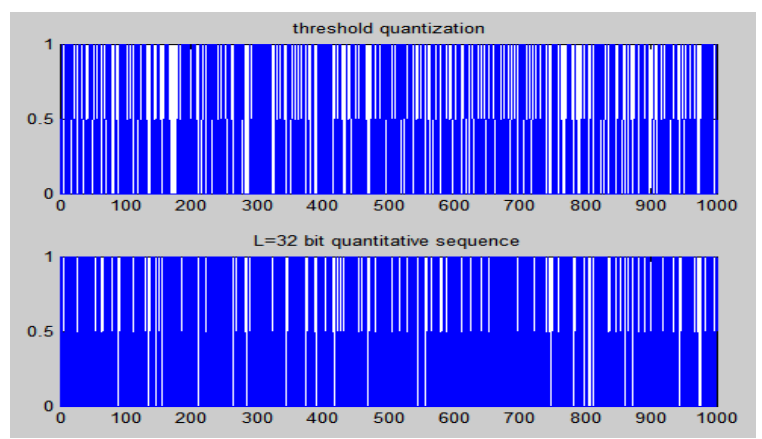

Fig. 3 Threshold quantization and bit quantization

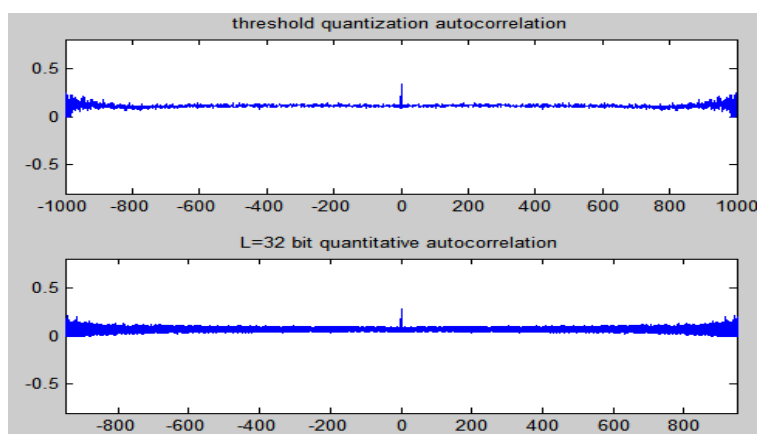

Fig. 4 Chebyshev quantitative autocorrelation 


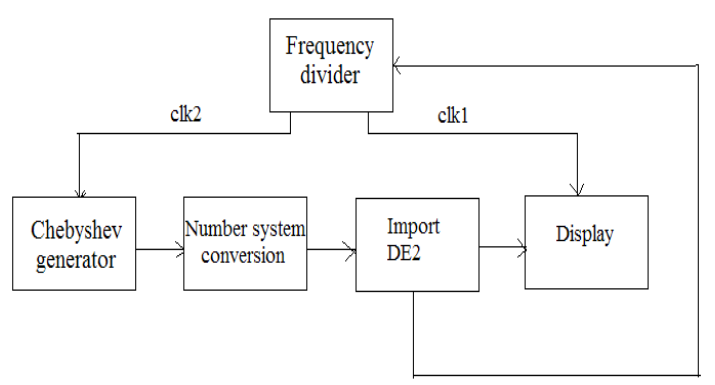

Fig. 5 Principle diagram

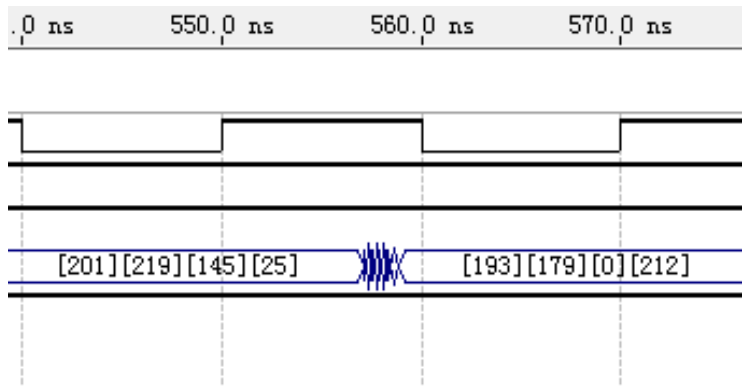

Fig.6 Waveform simulation

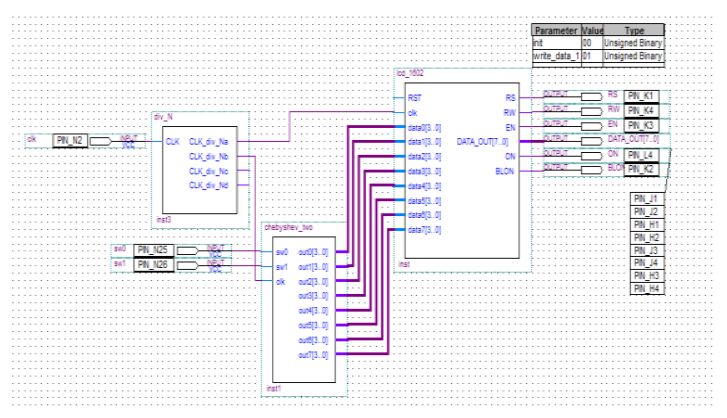

Fig. 7 Module diagram
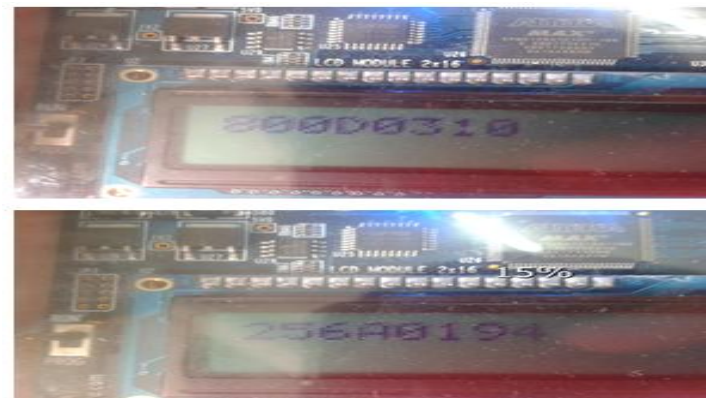

Fig. 8 Hexadecimal

\section{Conclusions}

In this paper, from the beginning of the pseudo-random sequence analysis, to explore the characteristics of the chebyshev generated random sequence. It has a deterministic and probabilistic, randomness and the differentiation of the relationship of unity of opposites. However, the pseudorandom sequence could not have this feature .And then, studies the real value relevance and chebyshev sequences after the results of several quantitative and the correlation of quantitative. This paper also gives a high order chebyshev sequences in the DE2 test method and results of quantitative numerical output. The better for us to study the higher order chebyshev sequences as well as the practical application of the future has great guiding significance and reference value.

\section{References}

[1] Yu Simin. Chaotic systems and chaotic circuit, principle, design and its application in communication. Xi'an University of electronic science and technology press, 2011. 4.

[2] Zhao Dongfeng, Tang Xiaomin, Ding Hongwei, Bao Liyong. Based on chebyshev-map the FPGA implementation of digital chaotic sequence. The 15th cross-strait conference on radio technology. Kunming, 2010, pp. 82-85.

[3] Liu PIng, Yan Chuan, Huang Xiangao. Improved chaotic spread spectrum sequence generation method based on Logistic mapping. Journal of communication, 2007, 28(2) pp. 134 140.

[4] Wang Hai, Hu Jiandong. Advanced logistic - map chaotic spread spectrum sequence. Journal of communications, 1997, 17 (8): pp 71-77.

[5] Gu Jingmin, Hong Xiaowen, Liang Tao.A modified Chebyshev chaotic sequence and its performance analysis. Journal of military communications, 2006, 27 (1): pp. 43-46.

[6] Tang Xiaomin, Zhao Dongfeng. FPGA Hardware Implementation of Digital Chaotic sequences Based on Chebyshev-map. Editor in Cheif Yang Yixian. Asia-Pacific Youth Conference on Communication Technology 2010 (APYCCT2010). USA: Scientific Research Publishing, 2010, pp. 740-743.

[7] Chens. Chaotic system circuit implementation and study. Nanjing: Nanjing University of Science \& Technology, 2009.

[8] Coulon, Martial. Roviras, Daniel. Multi-user receivers for a multipleaccess system based on chaotic system. Communications in Nonlinear Science and Numerical, 2011, pp 761-768.

[9] Chen Xi, Zhang Zhipeng. Communication system design based on Verilog HDL. Beijing: China water conservancy and hydropower press, 2009.

[10]Wu Jihua, Wang Cheng, Fan Lizhen. Alter the FPGA/CPLD design. Beijing: people's posts and telecommunications press, 2005, 7. 